\title{
Molecular Dynamics Simulation of Persistent Slip Bands Formation in Nickel-base Superalloys
}

\author{
Jianfeng Huang ${ }^{1 *}$ Zhonglai Wang ${ }^{2}$ Erfu Yang ${ }^{3}$ Don McGlinchey ${ }^{1}$ Yuanxin Luo $^{4}$ Yun Li $^{5}$ Yi Chen ${ }^{5}$ \\ ${ }^{1}$ School of Engineering and Built Environment, Glasgow Caledonian University, Glasgow G4 0BA, U.K. \\ ${ }^{2}$ School of Mechatronics Engineering, University of Electronic Science and Technology of China, People's Republic of China \\ ${ }^{3}$ Space Mechatronic Systems Technology Laboratory (SMeSTech), Department of Design, Manufacture and Engineering Management, James \\ Weir Building, University of Strathclyde, Glasgow G1 1XJ, U.K. \\ ${ }^{4}$ College of Mechanical Engineering, Chongqing University, Chongqing, People's Republic of China \\ ${ }^{5}$ College of Computer Science and Technology Dongguan University of Technology Songshanhu, Dongguan, Guangdong 523808 People's \\ Republic of China
}

\begin{abstract}
Persistent slip band (PSB) is an important and typical microstructure generated during fatigue crack initiation. Intensive work has been done to investigate the mechanisms of the formation of persistent slip bands since the 1950s when Wadsworth ${ }^{[1]}$ observed the fatigue fracture in copper. Simulations have indicated that PSBs formation during fatigue crack initiation related to the dislocation driving force and interaction. In this paper, a molecular dynamics (MD) simulation associated with embedded atom model (EAM) is applied on the PSBs formation in nickel-base superalloys with different microstructure and temperature under tensile-tensile loadings. Five MD models which different in microstructure(pure $\gamma$ phase and $\gamma / \gamma^{\prime}$ phase), grain orientation([ $\left[\begin{array}{lll}1 & 0 & 0\end{array}\right]\left[\begin{array}{lll}0 & 1 & 0\end{array}\right]\left[\begin{array}{lll}0 & 0 & 1\end{array}\right]$ and $\left.\left[\begin{array}{lll}1 & 1 & 1\end{array}\right]\left[\begin{array}{lll}1 & 0 & 1\end{array}\right]\left[\begin{array}{lll}1 & \overline{2} & 1\end{array}\right]\right)$ and simulation temperature $(300 \mathrm{~K}, 600 \mathrm{~K}, 900 \mathrm{~K})$ were built up in these simulations. Our results indicated that PSBs formed within the $\gamma$ phase by massive dislocations pile-up and propagation which can penetrate the grain. Also, the temperature will affect the material fatigue performance and blur PSBs appearance. The simulation results are in strong agreement with published experimental test result. This simulation is based on the author's previously work ${ }^{[2]}$.
\end{abstract}

Highlight:

1. Investigation of the PSB formation via molecular dynamics simulation with three different parameters

2. A new deformation and velocity combination controlled simulation conduct for the PSB formation.

3. High performance computing of PSB formation.

4. Systematic analysis of the PSB formation at the atomic scale in which the dislocation play a critical role.

Keywords: Persistent Slip Bands, Molecular Dynamics, Superalloys, Computational Simulation, EAM.

\section{Introduction}

Fatigue crack is a kind of mechanical fault which causes the material crash suddenly but without evidence in advance. Since this characteristic of fatigue, it has been the major fault of the industrial product or building structure. The first report of fatigue behaviour was in 1837 related to miner chain fracture caused by fatigue. Literally, fatigue is a kind of phenomena of crack propagation in the metal caused by the cyclic load for a long period of time. Fatigue is the most important factor of metal failure because it's unpredictable under working environment. There were many disasters in history caused by the fatigue failure of some working part. For example, the twice Comet airliner disasters occurred in 1954 lead to 56 people dead on board due to fatigue failure ${ }^{[3]}$. These crashes caused by a small crack generated by metal fatigue near the radio direction, situated in the front of the cabin roof.

Because of the serious effect of fatigue on the safety, numerous researches have been done since the middle of 19th century. Many theories about metal fatigue have been formalized and new ideas about fatigue life prediction have been published, most important, many advanced instru-

Regular paper

Manuscript received date; 05-01-2016

This work was supported by School of Engineering and Built Environment, Glasgow Caledonian University. ments have been invented to measure and examine fatigue crack.

As extensive researches of fatigue phenomena have been done, it is known that the general scheme of fatigue damage evolution in crystalline materials include three main phases from the engineering point of view - crack initiation period in which a macrocrack with several tenths of a millimetre formed, crack propagation period where the crack reached a critical length, and finally, the fracture ${ }^{[4]}$. Research indicated that the main reason of fatigue crack initiation is persistent slip bands, while others are because of voids or inhomogeneous in the material(subsurface crack initiation) or the roughness of the material surface(surface crack initiation). Experiment evidence indicated that small markers emerge on the specimen surface which is smooth at first after a cyclic load on the specimen for a period time. With the cyclic load increasing, the density of these markers increases accordingly. And also, these slip marker would occur again at the same area if the specimen continue under the fatigue test after surface polished. The reason is that the PSBs had already been formed in the material subsurface. And with the ongoing evolution of PSBs, it would extend to the material surface with the pattern of extrusion and intrusion again. Those extrusions and intrusions are named persistent slip markers (PSMs) and the inner parts of the material which caused the PSMs are so called the persistent 


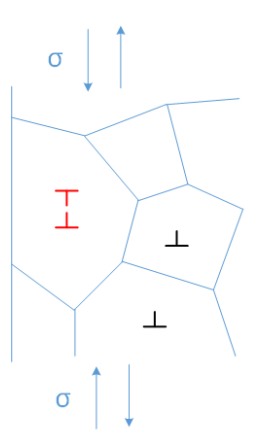

(a)

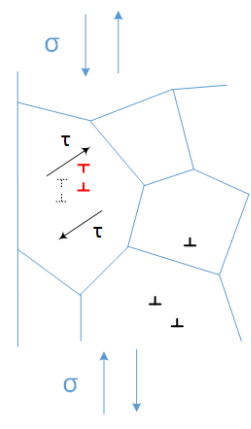

(b)

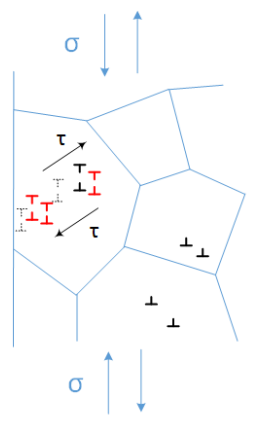

(c)

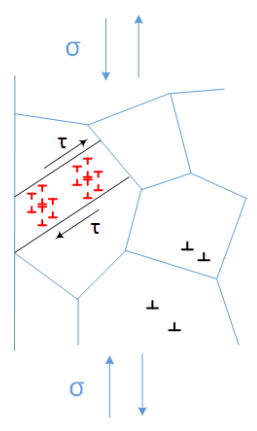

(d)

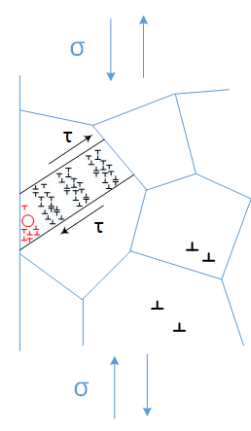

(e)

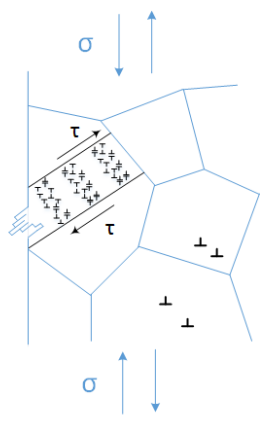

(f)

Fig. 1 Fatigue crack initiation from a) dislocation b) dislocations slip under critical resolved shear stress, c) dislocation blocked, d) low energy dislocation pathway and PSB formed, e) vacancy generated by dislocation annihilate, f) intrusion and extrusion formed crack initiation

slip bands.

Considering the importance of PSBs during the fatigue crack initiation, intensive works have been done to investigate the persistent slip bands formation and evolution. The common knowledge is PSB form after the initial hardening ceases with the dislocation matrix formed. For example, in copper single crystals the PSBs begin to appear when the material hardening stops and the cyclic stressstrain response saturation reaches ${ }^{[5]}$. As illustrated in Fig. 1, dislocations generated in the material under the cyclic load and they will slip along the crystalline slip system once the critical resolved shear stress reached. Some dislocations blocked by other dislocation, inhomogeneous, or grain boundary. With the cyclic load continue, the previous process repeated and lower energy pathway formed to allow the motion of dislocation continue along this way to form the dislocation wall. While, associated with the formation of the dislocation wall, dislocation matrix generated outside. At the same time, positive and negative dislocations interact with each other, once if close enough, they annihilate so that the vacancy nucleate. Finally, according to vacancies diffusion, the extrusions and intrusions will be formed in the grain boundary or material surface. During the PSBs formation, A.T. Winter ${ }^{[6]}$ found that the volume fraction of PSBs related to the disparate strain ratio distributing in PSBs and matrix. And the relationship of the shear plastic strain in different area is represented by the formula below:

$$
\gamma=(1-f) \gamma_{m}+f \gamma_{p s b}
$$

Where $\gamma$ is the material shear plastic strain and $\gamma_{m}, \gamma_{p s b}$ represent the shear plastic strain of matrix and PSBs respectively. And $f$ is the volume fraction of PSBs. Experiment revealed that PSBs are likely form at the range of plastic strain amplitudes of $1 \times 10^{-4} \leqslant \epsilon_{p a} \leqslant 1 \times 10^{-3}$ on individual cycled nickel polycrystalline grains having considerable different axial orientations ${ }^{[7]}$.

With the PSBs motivation and evolution, ladder-like dislocation wall perpendicular to the primary slip direction will be formed. Fig. 2 illustrates the typical unit PSB along the $\left[\begin{array}{lll}1 & 0 & 1\end{array}\right]$ direction on plane $\left(\begin{array}{lll}1 & 1 & 1\end{array}\right)$. The thickness of the slab normally $1 \sim 2 \mu \mathrm{m}$. The dislocation walls consist majority of edge dislocation dipole and the distance between the dislocation walls is regularly about $1.3 \mu \mathrm{m}^{[8]}$.

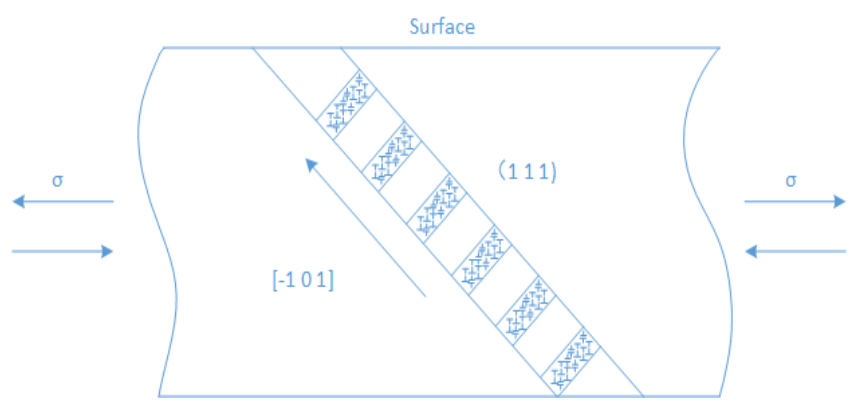

Fig. 2 Unit Persistent Slip Band diagram

When cyclic loads applied on the material continue, extrusion and intrusion finally emerge on the surface of the material. While dislocations motivation displays complex dynamics behaviours at this time. Among the dislocation movement, some dislocations exhibit the movability while others are the inhabitant, the first one can be called activator and the later is so called inhibitor. To evaluate the dislocation, a set of spatial and temporal based dislocation density functions $\rho_{m}(x, t)$ and $\rho_{i}(x, t)$, as known as the Walgraef-Aifantis model, provided by $[9,10]$. These dislocation density functions have two parts, one is to describe the activator dislocations density which is the domination. And the other part is to describe the inhibitor dislocation density which is a minor partition. The two functions are given below:

$$
\begin{gathered}
\frac{\partial \rho_{i}}{\partial t}=D_{i} \frac{\partial^{2} \rho_{i}}{\partial x^{2}}+g\left(\rho_{i}\right)-\beta \rho_{i}+\gamma \rho_{m} \rho_{i}^{2} \\
\frac{\partial \rho_{m}}{\partial t}=D_{m} \frac{\partial^{2} \rho_{m}}{\partial x^{2}}+\beta \rho_{i}-\gamma \rho_{m} \rho_{i}^{2}
\end{gathered}
$$

In this partial differential function, $D_{m}$ and $D_{i}$ donate the diffusion coefficient of both mobile and immobile dislocation separately. It is considered that the gradient of dislocation density of time is contributed by terms 1) $D_{i} \frac{\partial^{2} \rho_{i}}{\partial x^{2}}$, $D_{m} \frac{\partial^{2} \rho_{m}}{\partial x^{2}}$, the diffusion of immobile and mobile dislocations, separately 2) $\beta \rho_{i}$, the transformation of immobile dislocations to mobile dislocation, in which $\beta$ is a Heaviside-like parameter related to the applied stress. It plays the role of a bifurcation parameter at a certain level of stress. and 3) 
$\gamma \rho_{m} \rho_{i}^{2}$, the transformation of mobile dislocations to immobile dislocations. 4) $g\left(\rho_{i}\right)$, which only in the equation of immobile dislocation density, represents a function of the generation of immobile dislocation by the applied shear stress considered. In this model, it assumed the immobile dislocation diffusion coefficient was far less than the mobile dislocation diffusion coefficient because of the mobility of activator was much greater than that of inhibitor. This model reflected the characteristic of PSBs in dislocation generating, dislocation diffusion, and dislocation interaction. And it considered the PSBs formation because of the interaction between activator and inhibitor in which the activator was incapable of damping locally the growth of fluctuations of the inhibitor by the diffusion spreading. The limitation of this model was the independent of stress and temperature since only a few parameter related.

In this paper, our purpose is to provide an atomic model to investigate the unit PSB formation mechanism. We will introduce our molecular dynamic simulation method and related technician in section II and build up the atomic model at section III. Finally, we will analyse the simulation results in section IV, discuss and give the conclusion in section V and VI.

\section{Methodology}

Molecular dynamics is widely applied in chemical, material and biological science because of its special simulation ability on the study of the physical problem in atomic scale by solving a serial of ordinary differential functions, such as classical mechanics motion equations with Newton second law or the classical Hamilton function. Although MD is less precise than other simulation methods based on the quantum theory such as density functional theory (DFT), it is popular and well developed since the good performance. And also, the precise of the MD simulation results can be improved by adopting the potential between particles calculated by DFT. The fundamental of MD is to study the physical properties of the material by analysing the discrete particles trajectory which is calculated by solving these differential equations. Obviously, the molecular dynamics models consist of discrete particles, and besides, include their interactions potential which take action in a given ensemble with defined boundary conditions. It is known from the Newtons second law of motion that $F_{i}=m_{i} a_{i}$. And also the force can be derived from the gradient of potential function $\frac{\partial E_{t o t}}{\partial r_{i}}$ with respect to atomic displacements.

$$
F_{i}=\frac{\partial E_{t o t}}{\partial r_{i}}
$$

Normally, the $E_{\text {tot }}$ can be simply represented with a sum of pairwise interactions in which the famous one is LennardJones (LJ) potential ${ }^{[11]}$.

$$
E_{t o t}=\sum_{i} \sum_{j>i} \Phi\left(\left|r_{i}-r_{j}\right|\right)
$$

While within the crystalline metal, the better representation of the energy is the following embedded atom method model potential ${ }^{[12]}$ which is a many-body interaction between atoms associated with the function of the electron cloud in the metal.

$$
E_{t o t}=\frac{1}{2} \sum_{i, j(i \neq j)} \Phi_{S_{i} S_{j}}\left(r_{i j}\right)+\sum_{i} F_{S_{i}}\left(\bar{\rho}_{i}\right)
$$

In this EAM potential equation, the first term is the sum of all pair interactions between atoms similar with LJ potential, $\Phi_{S_{i} S_{j}}$ is a pair-interaction potential between atoms $i$ and $j$ in which have different atom types of $S_{i}$ and $S_{j}$.at positions $r_{i}$ and $r_{j}=r_{i}+r_{i j}$. The second term is the sum of embedding energy of all atoms in the system. Function $F_{S_{i}}$ denotes the energy of atom $i$, which depends upon the host electron density $\bar{\rho}_{i}$ at site $i$ induced by all other atom$\mathrm{s}$ of the system. Based on this method, G.P. Purja Puna and Y. Mishin ${ }^{[13]}$ published a developed Ni-Al system EAM potential which demonstrates a fairly good agreement with experimental and ab initio results for the formation energies of several other compounds of the Ni-Al system in 2009.

With the advantage of molecular dynamics simulation, many works have been deployed to research the nature of the material in the atomics level. And many post analysis methodologies have been applied for the case study. For example, Musazadeh and Dehghan ${ }^{[14]}$ studied the crack propagation behavior in nanocrystalline $\mathrm{Ni}$ which contains different shapes and types of second phases with $\mathrm{Pd}, \mathrm{Cu}, \mathrm{A}$, and Ag. Their MD simulation found that the short cylindrical shape exhibited a minority effect compared to the long cylinder impurity by analyzing the crack growth rate and strain energy release rate. In $2 \mathrm{D}$ plane case, the strain energy release rate $G$ is defined by:

$$
G=\frac{E W \varepsilon^{2}}{2\left(1-\nu^{2}\right)}
$$

where $E$ is Youngs modulus and $\nu$ is Poissons ratio, $W$ is the width of simulation box, $\varepsilon$ is the applied strain. Similar, in 2012, Paul White researched the fatigue crack growth in aluminum with the MD methods associated with isotropic linear elastic fracture mechanics (LEFMs). In this simulation, a cylinder atomic model with pre-notched crack containing nearly 3 million atoms simulated with three different potentials (MFMP99 ${ }^{[15]}, \mathrm{LEA}^{[16]}{ }^{[16}$, SKCFC11 ${ }^{[17]}$ ) potential) under a fatigue load which ratio was $R=0$. The significant difference between those potentials was the measured stable stacking fault energy $\gamma_{S F}$ which affects the extent of dissociation of dislocation and unstable stacking fault energy $\gamma_{U S F}$ which represents the energy barrier to

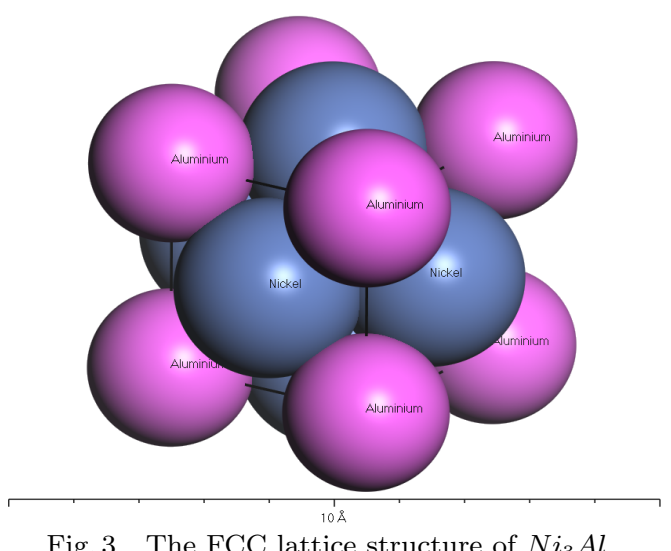

Fig. 3 The FCC lattice structure of $N i_{3} A l$ 
Table 1 The simulation model test cases setup

\begin{tabular}{|c|c|c|c|c|}
\hline & Benchmark & Test case 1 & Test case 2 & Test case 3 and 4 \\
\hline Model Size & \multicolumn{4}{|c|}{$500 \AA$ × $200 \AA$ x $40 \AA$} \\
\hline Composition & $\gamma / \gamma^{\prime}$ & $\gamma$ & $\gamma / \gamma^{\prime}$ & $\gamma / \gamma^{\prime}$ \\
\hline Direction & {$\left[\begin{array}{lll}1 & 1 & 1\end{array}\right]\left[\begin{array}{lll}\overline{1} & 0 & 1\end{array}\right]\left[\begin{array}{lll}1 & \overline{2} & 1\end{array}\right]$} & {$\left[\begin{array}{lll}1 & 1 & 1\end{array}\right]\left[\begin{array}{lll}\overline{1} & 0 & 1\end{array}\right]\left[\begin{array}{lll}1 & \overline{2} & 1\end{array}\right]$} & {$\left[\begin{array}{lll}1 & 0 & 0\end{array}\right]\left[\begin{array}{lll}0 & 1 & 0\end{array}\right]\left[\begin{array}{lll}0 & 0 & 1\end{array}\right]$} & {$\left[\begin{array}{lll}1 & 1 & 1\end{array}\right]\left[\begin{array}{lll}\overline{1} & 0 & 1\end{array}\right]\left[\begin{array}{lll}1 & \overline{2} & 1\end{array}\right]$} \\
\hline Temperature(K) & 300 & 300 & 300 & $600 / 900$ \\
\hline Cyclic Share Deformation Rate & \multicolumn{4}{|c|}{0.0005 along $\mathrm{Y}$ direction and velocity of $0.46-0.98$} \\
\hline Step/Step size & \multicolumn{4}{|c|}{1000 steps for each load or unload process ( $1 \mathrm{fs}$ for half cycle, 2 fs for whole cycle) } \\
\hline
\end{tabular}

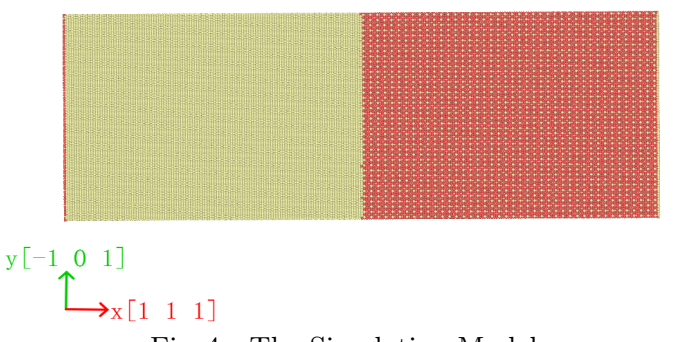

Fig. 4 The Simulation Model

dislocation nucleation. The simulation and energy analysis revealed that with lower stacking fault energy (SKCFC11), dislocation emission occurred faster than that with others potential, and material with lower unstable stacking fault energy appeared strong stability thus the formation of dislocation became difficult ${ }^{[18]}$.

Also, Po-Hsien Sung and Tei-Chen Chen ${ }^{[19]}$ studied the crack growth and propagation behavior in single crystal $\mathrm{Ni}$ by MD method associated with tight-binding potential. By analysis the von Mises stress and centrosymmetric parameter (CSP) distribution in the nanoribbon single crystal $\mathrm{Ni}$, the partial dislocations slips was observed at the crack tip in the close-packed (lllll) plane till material fracture occurring and critical stress in single crystal $\mathrm{Ni}$ follows the order

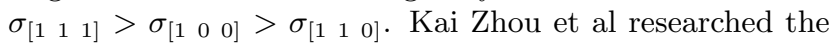
effect of grain size and shape on mechanical properties of nanocrystalline copper with MD simulation by comparing the some macro properties of the material such as stress and Youngs modulus ${ }^{[20]}$.

Besides the energy release rate analysis, stress - strain analysis and CSP analysis, Mao Wen et al ${ }^{[21]}$ developed a deformation index (DI) during their research of the hydrogen embrittlement phenomenon in single crystal $\mathrm{Ni}$ with EAM potential. The DI $\mu_{i}$ of atom $i$ is defined as:

$$
\mu_{i}=\frac{\max \left(\left|\overrightarrow{r i j}_{i j}-{\overrightarrow{i_{j j}}}^{0}\right|\right)}{|b|}
$$

where $\overrightarrow{r_{i j}}$ is the relative position vector of atom $i$ and $j$ and $\overrightarrow{r i j}^{0}$ is the vector in reference lattice, $b$ is the Burgers vector of $1 / 2\left[\begin{array}{lll}1 & 1 & 0\end{array}\right]$. With the DI analysis, the slip activities and dislocations can be traced and located immediately. Any lattice imperfections, torsion and structure changes can also be displayed clearly.

\section{Modelling}

To compare the effect of lattice orientation, phase and temperature on PSBs formation in Nickel alloy, three different group cases were studied in this paper. The first group includes two test cases, one with $\gamma$ phase pure $N i$, and another case, which was a benchmark test case, was $\gamma$ phase associated with $\gamma^{\prime}$ phase of $N i_{3} A l$. Both $\gamma$ and $\gamma^{\prime}$ phase were face-centered cubic (FCC) lattice and the lattice constant of $\gamma$ is $3.52 \AA$ and $\gamma^{\prime}$ was $3.572 \AA^{[22]}$. The FCC lattice structure of $N i_{3} A l$ was shown in Fig. 3. The lattice orientation in the simulation box was along direc-

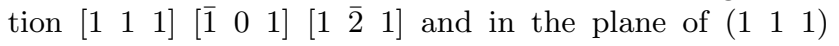
which was the close package plane and atoms tend to slip. The simulation box was about $520 \AA$ × $200 \AA$ × 40 $\AA$ (Fig. 4). The unconstrained misfit ${ }^{[23]}$ between the two regions of $\gamma$ and $\gamma$ was 0.015 in the combined phases case. The second group included two test cases all with $\gamma$ and $\gamma$ phase but the orientation was different, one was same as the test case in the first group, but another one was along $\left[\begin{array}{lll}1 & 0 & 0\end{array}\right]\left[\begin{array}{lll}0 & 1 & 0\end{array}\right]\left[\begin{array}{lll}0 & 0 & 1\end{array}\right]$ lattice direction. And the test cases in the final group was same as the benchmark test case in the first group but under the different temperature of $600 \mathrm{~K}$ and $900 \mathrm{~K}$ separately. Totally, nearly 300 thousand atoms created in all these simulation tests in which the grain size was about $10 \mathrm{~nm}$. This was a very fine grain size expected with high fatigue resistance. Periodic boundary condition was set on the $\mathrm{x} \mathrm{y} z$ directions which indicated the repeatable atoms topography along the directions. These simulations ran with Large-scale Atomic/Molecular Massively Parallel Simulator (LAMMPS) which is a parallel computation system. And the simulation result was visualized by OVITO(www.ovito.org). During the simulation of these designed test cases, at first, minimization of the system energy was run at the temperature of $300 \mathrm{~K}$. The equilibrium of the system would make the whole system be in a stable condition. After that, a deformation rate of 0.0005 in y direction was applied in the simulation area and uneven velocity range from 0.46 to 0.98 was applied on the different simulation groups along the $\mathrm{Y}$ and reverse Y direction for 1000 simulation steps separately with step time of $0.001 \mathrm{ps}$, and total simulation time in this load turn was $2 \mathrm{ps}$. The whole process repeated several times to apply the cyclic load on test systems. Simulations were performed by parallel computing with 60 cores on ARCHIEWeSt(www.archie-west.ac.uk) which is a high performance computer built with 3408 cores for distributed parallel and serial computing. It took 50 core hours for each test case.

\section{Simulation Result}

\section{1 $\gamma^{\prime}$ phase effect on PSB formation}

The precipitate second phase is the mainly strengthen factor in the nickel base superalloy manufactory. It plays the role of barrier against dislocation motion thus enhance the yield strengthen of superalloys. Generally, the strengthening effect depends on the mechanical properties of the particles precipitated in the material. Based on this, there 
are two kinds of mechanism in the second phase strengthen theory: the first mechanism is that dislocations cutting through particles if the particle softer than the base element. And the another one is dislocations pass through the particle by bowing and the dislocation loops are formed after them, also this is known as Orowan strengthening ${ }^{[24]}$. The first law is:

$$
\tau=\frac{\pi r \gamma}{b L}
$$

where $\tau$ represents material strength, $r$ is the second phase particle radius, $\gamma$ is the surface energy, $\boldsymbol{b}$ is the Burgers vector, and $L$ is the spacing between pinning points. This governing equation shows that the strength is proportional to $r$ which indicates that as the size of the second phase particles increases, the particle impede dislocation movement and it becomes increasingly difficult for the particles to cut through the material. In other words, the strength of a material increases with increasing $r$.

While in Orowan strengthening mechanism, the greater size particles can't be cut but only by bowing of dislocations. The equation for this model is:

$$
\tau=\frac{G \boldsymbol{b}}{L-2 r}
$$

where $\tau$ is the material strength, $G$ is the shear modulus, $\boldsymbol{b}$ is the magnitude of the Burgers vector, $L$ is the distance between pinning points, and $r$ is the second phase particle radius. Considering the two mechanisms together, it reveal$\mathrm{s}$ that the second phase particles have a critical size with which the material will exhibit maximum yield strength. Besides that, the second phase also plays a role in enhancing the fatigue resistance. For example, in Nickel alloy, a big size of impurity second phase had a strong effect to prevent the crack growth. And if in the case the alloying elemen$\mathrm{t}$ atom weight and lattice constant were greater than $\mathrm{Ni}$, less energy released rate was detected which means some elements such as Pd contribute to the strengthen of Nickel alloy than other elements such as $\mathrm{Cu}$ and $\mathrm{Au}$.

In our simulation, we compared the effect of second phase $\gamma^{\prime}$ with the pure $\gamma$ phase in the simulation test case 1 which showing in Table 1. By introducing the $\gamma^{\prime}$ phase, the lattice parameter is different than $\gamma$ phase since the present of Aluminium atoms. This misfit introduced by the second phase could cause antiphase boundary(APB) which increase the alloys tensile strength. The phase boundary plays a key role to obstacle the dislocation motion between the phase thus increases the strength. To quantify the occurrence of defects in this simulation, the microstructure of the material was analyzed by adaptive common neighbor analysis $(\mathrm{CNA})^{[25]}$ and centrosymmetric parameters $(\mathrm{CSA})^{[25]}$. In single $\gamma$ phase with the same strain rate and temperature, the dislocations were formed along the strain direction and scatter among the material. From the centrosymmetric parameter picture, the parameter $\mathrm{P}$ was greater than $8.3 \AA^{2}$ in an FCC lattice indicated an intrinsic stacking fault. The red particles in Fig. 5 a) shows the intrinsic stacking fault in pure single crystalline nickel generated along the $\left[\begin{array}{lll}\overline{1} & 0 & 1\end{array}\right]$ direction in ( $\left.\begin{array}{lll}1 & 1 & 1\end{array}\right)$ plane. From Fig. 5 b), CNA analysis in the case indicate the pure FCC structure was only $81.2 \%$

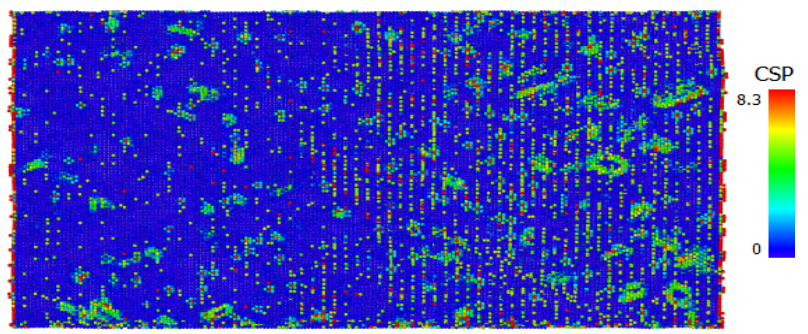

(a)

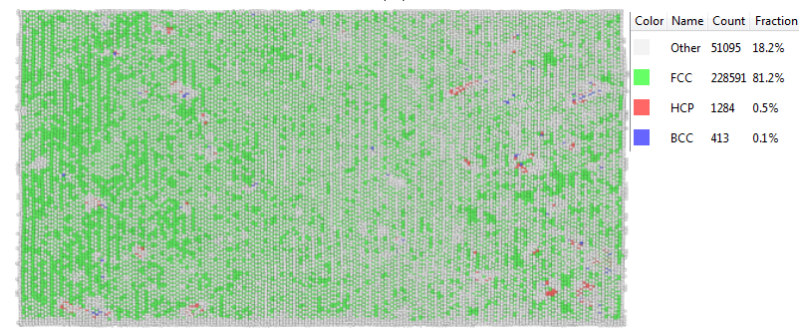

(b)

Fig. 5 The CSP and CNA contour plot of $\gamma$ phase pure nickel under fatigue load ratio ( $\mathrm{min}$ load $/ \mathrm{max}$ load) $=0$ after $40 \mathrm{ps}$. a) is CSP display of $\gamma$ phase pure nickel in which dislocation loop and dislocation slip along [ $\left[\begin{array}{lll}1 & 0 & 1\end{array}\right]$ direction. $b$ ) is CNA display of $\gamma$ phase pure nickel which shows the FCC structure is $81.2 \%$

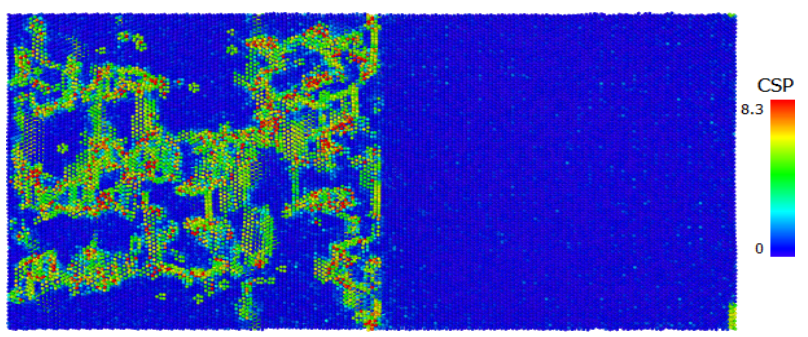

(a)

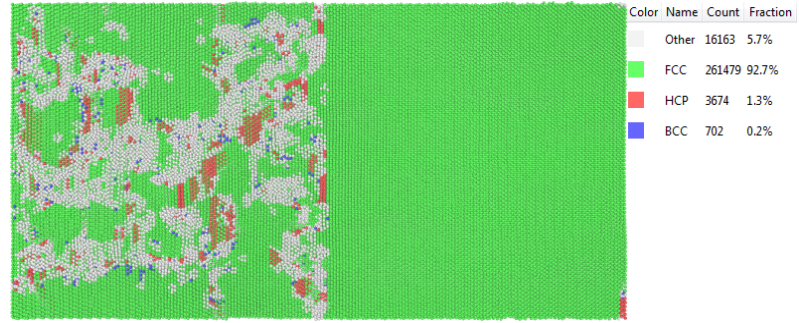

(b)

Fig. 6 The CSP and CNA contour plot of $\gamma / \gamma^{\prime}$ phase under fatigue load ratio $=0$ after 40 ps. $a$ ) is CSP display of $\gamma$ phase in which dislocation pile-up occurs and dislocation slip along [101] direction. but few dislocation in $\gamma^{\prime}$ phase $b$ ) is CNA display of

$\gamma$ and $\gamma^{\prime}$ phase which shows the FCC structure is $92.7 \%$

while HCP was only $0.5 \%$. That mean few intrinsic stacking fault in this system during deformation. But this dislocation behaviour was totally different against that occurred in $\gamma / \gamma^{\prime}$ phase test case. When investigating the trajectories of particles in $\gamma / \gamma^{\prime}$ phase as Fig. 6 a) displayed, evidently, the $\gamma$ phase was the activity basic matrix which likely to absorb energy by dislocations generation ${ }^{[26]}$ in the surface while $\gamma$ phase was the strength and stable participation which had less distortion during strain increasing in room temperature. Dislocations were likely to nucleate and slip 

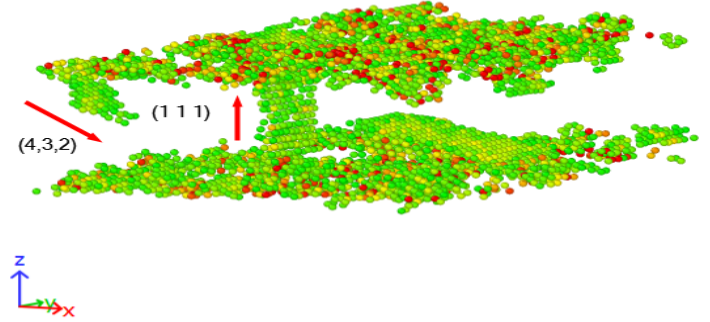

Fig. 7 dislocation slip in (4 $\left.\begin{array}{lll}4 & 2\end{array}\right)$ and (1 111 ) plane which penetrate the grain in $\gamma$ phase and nucleated in the boundary of $\gamma / \gamma^{\prime}$ phase

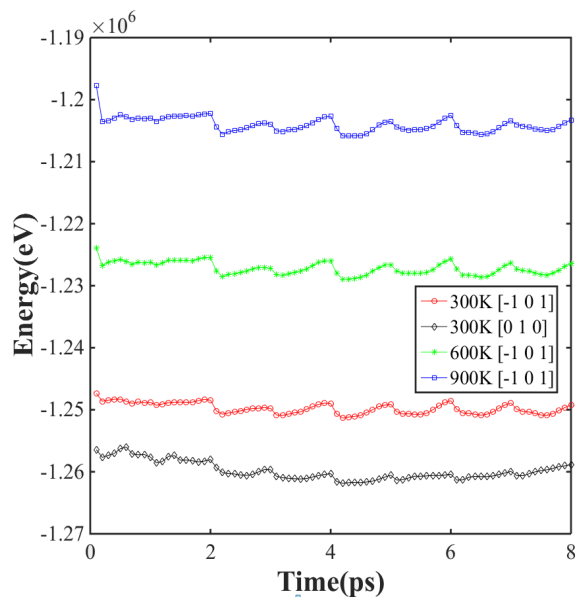

(a)

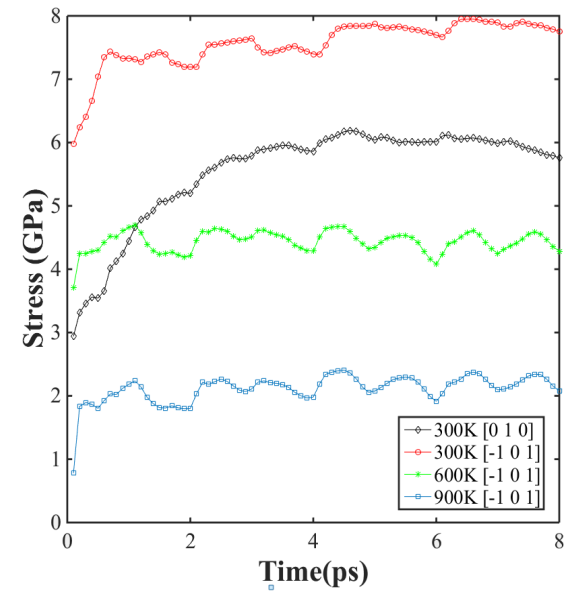

(b)

Fig. 8 Diagram of energy and mean stress in different case

along the y direction in $\gamma$ phase. And coupled with the CNA in Fig. 6 b), the atoms which was not belong to FCC structure were nearly $7.3 \%$ in the whole system that demonstrate the dislocation volume. At the same time, some dislocations propagate and penetrate into the body by the slip in the plane $\left(\begin{array}{lll}4 & 3 & 2\end{array}\right)$ and $\left(\begin{array}{lll}1 & 1 & 1\end{array}\right)$. It was notable that, partial dislocations nucleated in the boundary of $\gamma$ and $\gamma^{\prime}$ phase which demonstrated a clear view of the phase discrimination (Fig. 7).

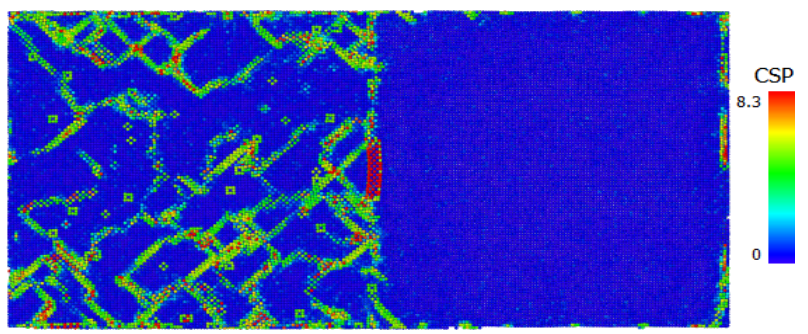

(a)

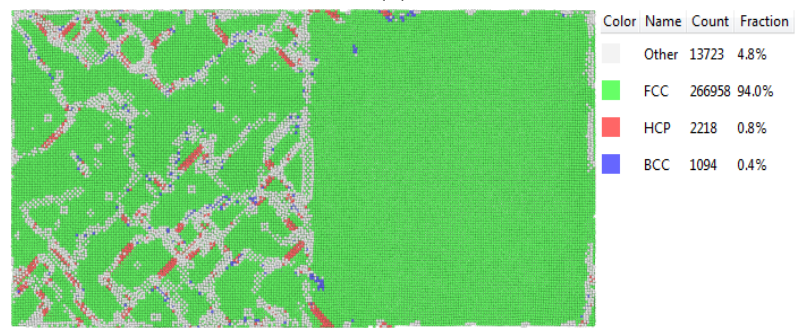

(b)

Fig. 9 The CSP and CNA contour plot of $\gamma / \gamma^{\prime}$ phase under fatigue load ratio $=0$ along $\left[\begin{array}{lll}1 & 0 & 0\end{array}\right]$ directions after 40 ps. $a$ ) is CSP display of $\gamma / \gamma^{\prime}$ phase in which dislocation slip along $45 \mathrm{deg}$ directions. But few dislocation in $\gamma^{\prime}$ phase $b$ ) is CNA display of $\gamma / \gamma^{\prime}$ phase which shows the FCC structure is $94.1 \%$

\subsection{The effect of orientation}

From the atomic scale's point of view, the microstucture of metal is anisotropy and the elastic moduli is notably depending on the relation of grain or lattice orientation to the testing direction. The lattice orientation is one of the three significant microstructure variables (others two are grain size and shape). All experiments ${ }^{[27,28]}$ and simulations ${ }^{[29,30]}$ indicates that mechanical properties of metal, especially of the fracture behaviour, related to the crystal orientation. That's because of slip systems of crystal lattice is orientation related.

In FCC structure, there are 12 slip systems which are composed by 4 primary slip planes of $\left\{\begin{array}{lll}1 & 1 & 1\end{array}\right\}$ associated with 3 different directions of $\langle 1 \overline{1} 0\rangle$. For the symmetry of FCC crystal structure, we only considered the primary slip plane $\left(\begin{array}{lll}1 & 1 & 1\end{array}\right)$ with direction $\left[\begin{array}{lll}1 & 0 & 1\end{array}\right]$ which used in the benchmark test case. It's notable that the dislocation slipped and accumulated along the $\left[\begin{array}{lll}1 & 0 & 1\end{array}\right]$ direction in the $\gamma / \gamma^{\prime}$ phase boundary in primary slip plane $\left(\begin{array}{lll}1 & 1 & 1\end{array}\right)$ since the slip plane was parallel to the phase boundary. Also the high energy barrier of the phase boundary repelled the dislocations activity near the phase boundary. While dislocations in the $\gamma$ phase body, dislocation slip activity occurred not in the this prime slip plane but only in the other one $\left(\begin{array}{lll}\overline{1} & 1 & 1\end{array}\right)$.

When change the lattice parameter from [lll 111$]\left[\begin{array}{lll}1 & 0 & 1\end{array}\right]$ $\left[\begin{array}{lll}1 & \overline{2} & 1\end{array}\right]$ to $\left[\begin{array}{lll}1 & 0 & 0\end{array}\right]\left[\begin{array}{lll}0 & 1 & 0\end{array}\right]\left[\begin{array}{lll}0 & 0 & 1\end{array}\right]$ of lattice orientation, we found the only difference with the benchmark case was that the dislocation slips along the 45 degree directions (Fig. 9). By investigating this simulation process in detail, we discovered, firstly, the intrinsic faults in the surface were introduced by the dislocation slips along the $\left[\begin{array}{lll}1 & 1 & 0\end{array}\right]$ and $\left[\begin{array}{lll}1 & \overline{1} & 0\end{array}\right]$ direction within the surface, and then, the formation of the dislocation line in these directions. With the cyclic loading continued, dislocation slip began to occur in planes of $\left(\begin{array}{lll}\overline{1} & 1 & 1\end{array}\right)\left(\begin{array}{lll}\overline{1} & 1 & \overline{1}\end{array}\right)$ and $\left(\begin{array}{lll}1 & 1 & 1\end{array}\right)$ and infiltrated into the grain 
center. Once the dislocation slips crossed each other in the body, the intrinsic stacking fault was formed with the cyclic load increased. By comparing the stress (Fig. 8) in the test cases, it seemed that even in the test case with dislocation volume fraction was similar with that in benchmark test case, the mean stress with the same strain was much lower than that in benchmark since there was few dislocations pile-up with the cyclic load applied. Also in the benchmark test case, because of the load applied was perpendicular to the plane of $\left(\begin{array}{lll}1 & 1 & 1\end{array}\right)$ which was the closest package plane in FCC structure and caused strain along [ $\left[\begin{array}{lll}1 & 0 & 1\end{array}\right]$ direction, then greater magnitude of stress was needed to generate the same $\operatorname{strain}^{[31]}$.

\subsection{Temperature effect}

Temperature has a critical effect on superalloys in various aspects. As known in crystal solid, the atoms vibrate around their equilibrium positions. When the temperature increased, the vibration amplitude increases while frequency changes minor. Thus, the average interatomic distance and lattice parameters increase according to elevated temperature. This means the temperature will affect the nanostructure of the material. Besides, the distribution of atoms is different against different temperature level. The difference of atoms distribution will cause the different lattice structure formatted. Consider the Ni-base alloy, with temperature increase, the strengthen phase of $\gamma^{\prime}$ will slightly decreased from the maximum volume of fraction. What$\mathrm{s}$ more, the vacancies formation and dislocation motion in metal will be affected by temperature. For example, in BCC metals the dominant slip plane is along $\left\{\begin{array}{lll}2 & 1 & 1\end{array}\right\}$ when temperature below a quarter of melting point $\left\{\begin{array}{lll}3 & 2 & 1\end{array}\right\}$, while along when temperature is above previous one but below half of melting point. Definitely, this will change the deformation behavior of superalloys at elevated temperature.

High temperature fatigue always accompanies with creep behavior which caused by oxidization in the material surface. But, in this simulation, it only reflected the material subsurface microstructure changing. So the oxidization behavior was not included in this research scope. In this group test cases, with temperature increasing, thermal activation enhanced dislocations motivation, consequently, the dislocations became more homogeneous and dislocation density increased sharply. This could be shown in the last group test cases simulated under the elevated temperature at $600 \mathrm{~K}$ and $900 \mathrm{~K}$ separately (Fig. 10, 11).

With the temperature increasing, the system became more unstable by the mean stress decreased because of system energy increased dramatically as Fig. 8 a) shows. And also some defects of $\gamma^{\prime}$ phase which were scattered also observed in these case but not at room temperature. It was notable that, the dislocations and defects in $\gamma$ phase tend to pile up in the boundary of $\gamma / \gamma^{\prime}$ phase. Since the bonding energy decreases in high temperature which leads to atoms oscillating at a higher frequency and speed up, microstructure distortion became easier than that in room temperature. But at this situation, the intrinsic stacking fault was hard to form since the recovery mechanisms of $\gamma / \gamma^{\prime}$ at very high temperature. From the CSA and CNA graphic (Fig. 11 ) at $900 \mathrm{~K}$, we found the number of crystalline defects had

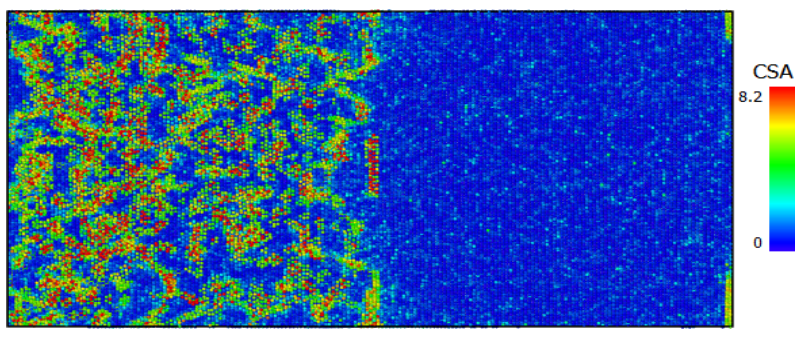

(a)

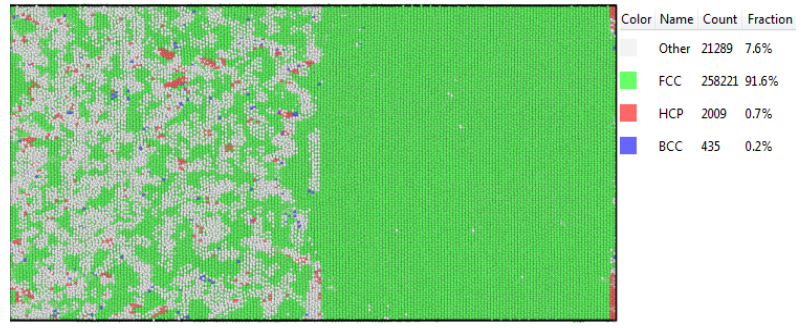

(b)

Fig. 10 The CSP and CNA contour plot of $\gamma / \gamma^{\prime}$ phase under fatigue load ratio $=0$ during temperature $600 \mathrm{~K} a$ ) is CSP display of $\gamma / \gamma^{\prime}$ phase $b$ ) is CNA display of $\gamma / \gamma^{\prime}$ phase which shows the FCC structure is $91.6 \%$

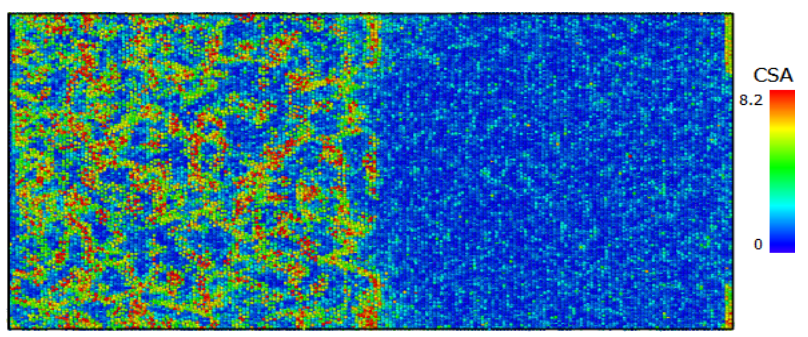

(a)

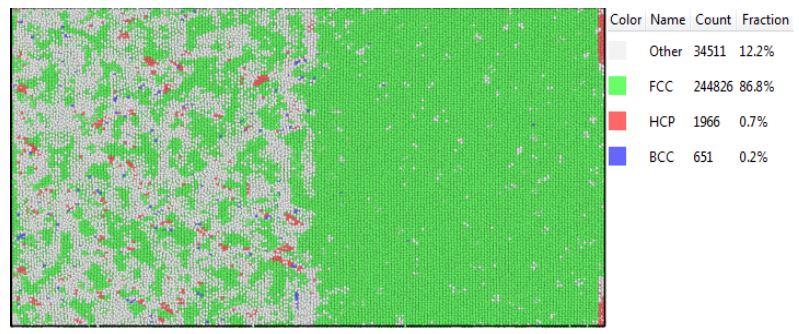

(b)

Fig. 11 The CSP and CNA contour plot of $\gamma / \gamma^{\prime}$ phase under fatigue load ratio $=0$ during temperature $900 \mathrm{~K} a$ ) is CSP display of $\gamma / \gamma^{\prime}$ phase $b$ ) is CNA display of $\gamma / \gamma^{\prime}$ phase which shows the FCC structure is $86.8 \%$

raised up by comparing the pure FCC structure (86.8\%), but few of them piled up in the material body. The agreement with the experimental result (Fig. 12) of Nimonic 80A indicates dislocations motivation under high temperature was active very much. And also with the reference from the mean stress graph, it revealed that, to generate such same strain, the stress turned to be much smaller rather than other situations. 

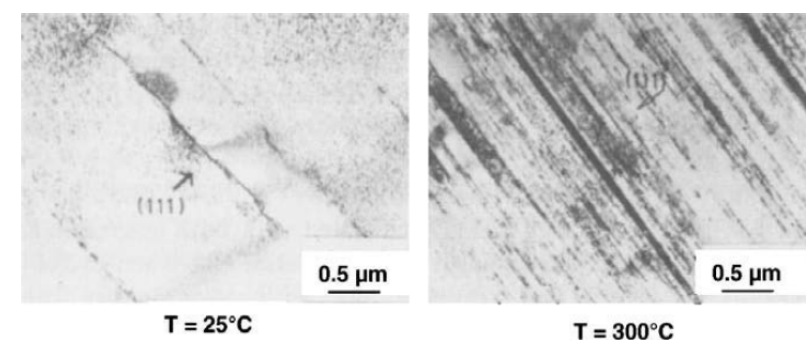

$\mathbf{T}=500^{\circ} \mathrm{C}$

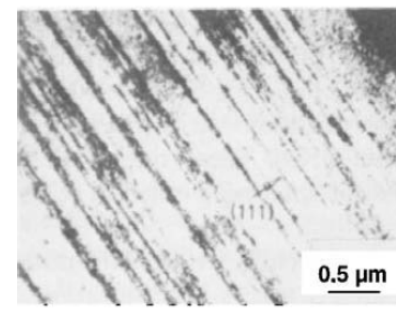

$\mathbf{T}=300^{\circ} \mathrm{C}$

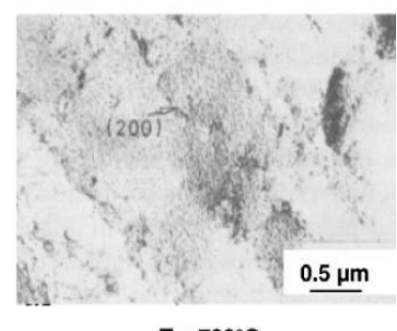

Fig. 12 Our simulation results have good agreement with the experiment results in [27], which indicated slip band density as a function of temperature for Nimonic $80 \mathrm{~A}$ tested at a plastic strain range of $0.15 \%$.

\section{Discussion}

By comparing the microstructure statistic chart(Fig. 13), it was evident that the volume fraction of perfect FCC lattice particles in benchmark and test case 2 were much greater than that of other test cases. This meant that the material which with the precipitated second phase strengthening will withstand less distortion under the load which perpendicular to primary slip plane in room temperature against other cases. While in test case 1 , the volume fraction of FCC perfect lattice percentage is the lowest among all tests which indicated that the $\gamma^{\prime}$ phase was the most important strengthen phase in Nickel alloys. It increased the fatigue resistance of Nickel alloys significantly. The data of test case 2 indicated that the orientation of external load was an important factor but had less effect against the material composition. The temperature's effect was notable also but not as strong as compared to the effect of precipitated second phase.

The reason caused that behaviour of superalloys in these simulations was stated below:

a), The microstructure of superalloys is considerable on the present of $\gamma^{\prime}$ phase which is a precipitated strengthen phase in Nickel superalloys, the misfit of the lattice structure between the $\gamma / \gamma^{\prime}$ phase boundary increased the phase energy and play the role of stability in the alloying system. Also, the ordered of $\mathrm{Ni}_{3} \mathrm{Al}$ structure contribute to the high performance of Nickel superalloys by considering the significant degree of directional and covalent bonding between $\mathrm{Ni}$ and $\mathrm{Al}$ atoms. From the EAM potential which applied in this study as shown in Fig. 14, the $\mathrm{Ni}-\mathrm{Al}$ bond energy is much lower than the pure $\mathrm{Ni}-\mathrm{Ni}$ or $\mathrm{Al}-\mathrm{Al}$ system. Since Gibbs free energy is directly proportional to the atomic bonding energy, the $\gamma^{\prime}$ phase free energy is less than pure $\mathrm{Ni}-\mathrm{Ni}$ or Al-Al system. As a result, the $\gamma^{\prime}$ is the stabilized phase in Nickel superalloys.

b), The orientation of external load is a stimulate of the slip system in the alloy system. With the different

\section{Perfect/Defect particles percentage}

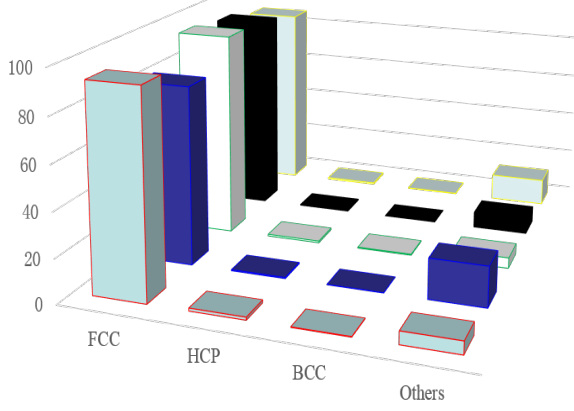

Benchmark Model $\gamma / \gamma^{\prime},\left[\begin{array}{lll}1 & 1 & 1\end{array}\right]\left[\begin{array}{lll}-1 & 0 & 1\end{array}\right]\left[\begin{array}{lll}1 & -2 & 1\end{array}\right], 300 \mathrm{~K}$

-Model 1 Different $\gamma$ phase

DModel 2 Different orientation [1 00$]\left[\begin{array}{lll}0 & 1 & 0\end{array}\right]\left[\begin{array}{lll}0 & 0 & 1\end{array}\right]$

- Model 3 Different temperature 600K

Model 4 Different temperature 900K

Fig. 13 The statistic plot of volume fraction of different microstructure particles according to different test cases

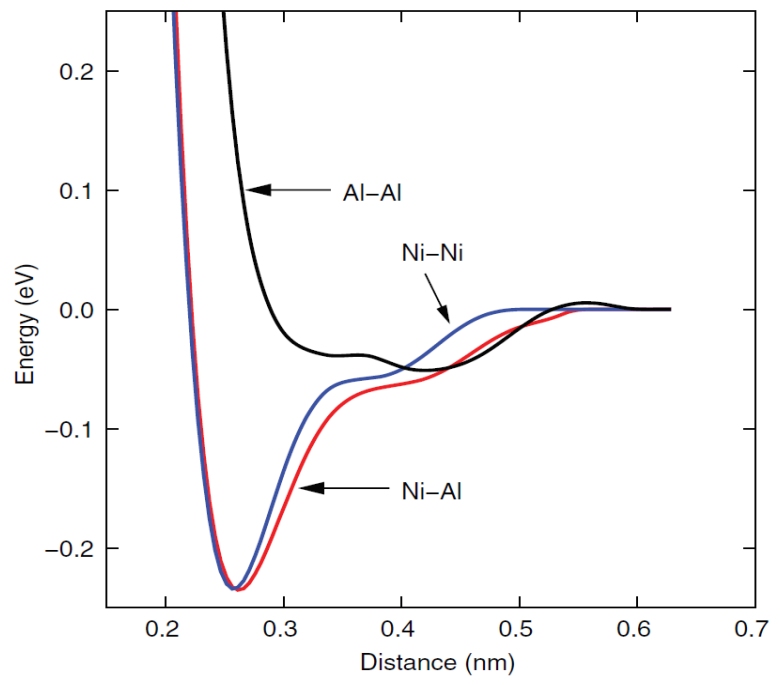

Fig. 14 Bond energy of the EAM potential presented in the effective pair format. courtesy of [13]

orientation, the number of active primary slip plane is different thus result in the mechanical properties changes in the atomic level. In the benchmark test case, there are 2 active slip systems in the plane of $\left(\begin{array}{lll}1 & 1 & 1\end{array}\right)$ along the two different directions according to the external loading. While in the test case 2 , there are more than 4 active slip systems which were activated in all primary slip planes in the $\gamma$ phase since the external loading direction was not perpendicular to them but with angles. Finally, dislocation activities presented on all these slip planes and crossed each other, partially, rectangle dislocation loop evolved once them can't cross each other.

And also, since the $\gamma / \gamma^{\prime}$ phase orientation is in the same, the phase boundary is more likely parallel to the primary slip plane in the benchmark test case. The phase boundary also plays a role of to prevent the penetration of slip activity on the phase boundary and increased the stress response according to the same strain. 


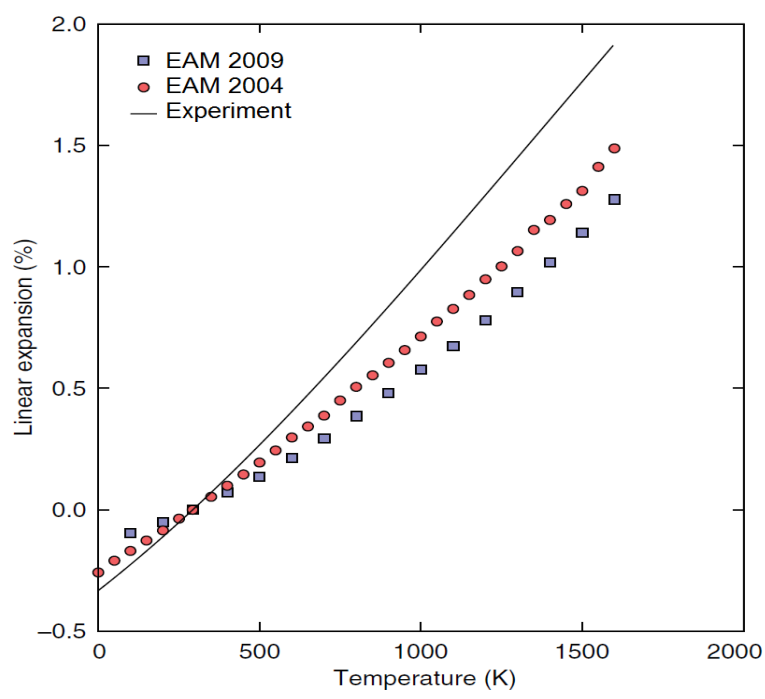

Fig. 15 Linear thermal expansion (\%) of $\mathrm{Ni}_{3} \mathrm{Al}$ relative to room temperature $(293 \mathrm{~K})$ computed with the new EAM potential in comparison with the EAM-2004 potential ${ }^{[28]}$ and experimental data ${ }^{[29]}$. Courtesy of [13]

c), Atoms diffusion activity increased according to the elevated temperature and the recrystallization of grain occurred. The linear thermal expansion data of $\mathrm{Ni}_{3} \mathrm{Al}$ was shown in Fig. 14 and compared with another EAM potential which provided by Mishin ${ }^{[28]}$ and experimental data $^{[29]}$. Since the thermal expansion factors in this EAM potential was computed by Monte Carlo which not included the quantum effects that caused discrepancies at low temperature $^{[13]}$, the expected result was less than the experimental result. This would affect our simulation result in the stress and strain which expected greater than current. Despite this negative effect, our result at elevated temperature also was also considerable because it reflect the truth that the stress at high temperature decreased according to the strain increasing by comparing the stress and energy graph in Fig. 8. But the dislocation interaction such as slip, glide, jog, and climb et. al. were not notable in this simulation result. From the CNA collection data were shown in Fig. 13, it indicated that the pure FCC structure decreased yet which means that the lattice torsion behaviour was stimulated by the temperature was much more considerable than the lattice orientation. If regarding the effect of the $\gamma^{\prime}$ strengthen phase, the temperature's effect was not so much critical to the material properties.

According to the discussion aforementioned, we can give conclusion in the following chapter.

\section{Conclusion}

From the MD simulation of Nickel alloy under different situations, our conclusions can be stated and it seems that the simulation results agreed with the experimental results:

$1, \gamma^{\prime}$ phase is the strengthening phase in Nickel alloy. During all of the simulation MD case, $\gamma^{\prime}$ phase is under few deformation, the PSB ladder like dislocation mostly occur in $\gamma$ phase. Dislocations tend to pile-up and penetrate into $\gamma$ phase which contribute to the fatigue crack initiation in Nickel alloy.

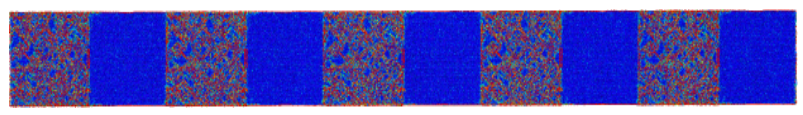

Fig. 16 Unit PSB formation by repeated boundary condition

2, Dislocation slip is the main activity during PSB formation. The dislocation slip behavior is observed in all test cases. This slip activity is more related to the temperature which cause system bond energy decrease. Once intrinsic stacking fault is formed in a material, dislocations tend to pile up in the close package plane in low temperature. With temperature increased, the dislocation has difficult to pin up because of the recovery mechanisms.

3 , A different orientation have the different result of cyclic loading response. Simulation results indicate if the load is perpendicular to close package plane, dislocations will pile up and restrict the dislocation motivation and then enhance the fatigue resistance of the material.

Because of spatial and temporal limitation of MD simulation, it is difficult to simulate the whole PSBs formation mechanism in tiny atomic structure, but with the periodic boundary condition, we have repeated the simulation and it indicates the ladder like structure is formed during fatigue load as on Fig. 15. Our future work will concentrate on the simulation of fatigue crack initiation within multicrystalline nickel-base alloy and compare with the experimental investigation.

\section{Future work}

There are still have plenty work to be finished to discover the PSBs activity and evolution in Nickel superalloys in the future since this is our first simulation for the PSBs. Four tasks are on our future work plan list. First, we will focus on the dislocation interaction which is a dominated phenomenon during the PSBs formation and to understand the mechanism of PSBs initialization in the atomic scope. To achieve this, a temperature related dislocation energy model based on Peierls-Nabarro model will be integrated into LAMMPS to enhance the EAM potential and to discover the dislocation motion and interaction in high temperature because of the shortage of the EAM potential in high temperature. Second, other factors like grain size and grain boundary are also the important parameter which should be considered during the PSBs formation. The polycrystalline model could be built up by integrating the Voronio $^{[30]}$ algorithm into LAMMPS modelling. The grain size and orientation could be decided by introducing a random factor generated by computational intelligence algorithm $^{[32]}$ which can simulate the nature of superalloys heat treatment and processing. And then, we will consider the other composite and microstructure such as Chromium, Cobalt because the two alloying elements have the magnificent volume fraction in Nickel superalloys. For example, in Inconel 740, the volume fraction of $\mathrm{Cr}$ and $\mathrm{Co}$ is in the range of $23.525 .5 \%$ and $1822 \%$ separately, while the volume fraction of other elements is less than $3 \%$. Also, the two elements are the strengthen factor in Nickel superalloys in which the strengthen phase could be $100 \%$ of $\gamma^{\prime}$ phase with Ni-Cr25\% when the temperature is above $800{ }^{\circ} \mathrm{C}^{[33]}$. To optimize these parameters during the simulation, a de- 
cision process with a state-of-the-art computational intelligence aided design (CIAD $)^{[34]}$ framework will be deployed in the prototype simulation system. Finally, the fatigue experiment with the fatigue crack initiation detection by jointed acoustic emission method and scanning electron microscopy will be finished in the future to validate our simulations result.

\section{Acknowledgement}

The authors would like to acknowledge the scholarship award to the first author provided by the SCHOOL OF ENGINEERING AND BUILT ENVIRONMENT, GLASGOW CALENDONIAN UNIVERSITY. And this work was also partially supported by National Natural Science Foundation of China (Grant No. 51405044, 51105061 and 11472075). Finally, partial results were obtained using the EPSRC funded ARCHIE-WeSt High Performance Computer (www.archie-west.ac.uk). EPSRC grant no. $\mathrm{EP} / \mathrm{K} 000586 / 1$. The author thanks the support provided by UNIVERSITY OF STRATHCLYDE.

\section{References}

[1] N.J. Wadsworth, N. Thompson Observations on the fatigue fracture of copper, The London, Edinburgh, and Dublin Philosophical Magazine and Journal of Science: Series 7, vol. 45, issue 361, pp. 223-224, 1954.

[2] J. Huang, Z. Wang, Y. Luo, Y. Li, E. Yang and Y. Chen, Computational investigation of superalloy persistent slip bands formation, Automation and Computing (ICAC), 21st International Conference on, Glasgow, 2015, pp. 1-6. doi: 10.1109/IConAC.2015.7313999

[3] R. J. Atkinson, W. J. Winkworth and G. M. Norris, Behaviour of Skin Fatigue Cracks at the Corners of Windows in a Comet I Fuselage No. 3248, HER MAJESTY'S STATIONERY OFFICE, London, Jan, 1962. (Report)

[4] I, Milne, R.O. Ritchie, B. Karihaloo Comprehensive Structure Integrity, 4.01 Cyclic Deformation, Crack Initiation, and Low-Cycle Fatigue, vol. 4. Elsevier. pp. 7.(Book style)

[5] S. X. Li, Y Li, G. Y Li, J. H. Yang, Z. G. Wang and K. Lu The early stages of fatigue and evolution of persistent slip bands in a copper single crystal, Phil. Mag. A vol. 82, issue 5, pp. 867-883, 2002.

[6] A.T. Winter, A model for the fatigue of copper at low plastic strain amplitudes, Phil. Mag, vol. 30, issue 4, pp. 719738, 1974.

[7] C. Buque. Persistent slip bands in cyclically deformed nickel polycrystals, International Journal of Fatigue, vol. 23, issue 6, pp. 459-466, July 2001.

[8] P. Lukáš, L. Kunz, Role of persistent slip band in fatigue, Phil. Mag. vol. 84, issue 3-5, pp. 317-330, 2004.

[9] D. Walgraef and E.C. Aifantis, On the formation and stability of dislocation patterns-III: Three-dimensional considerations, International Journal of Engineering Science, vol. 23, issue 12, pp. 1365-1372, 1985.

[10] M.V. Glazov and C. Laird, Size effects of dislocation patterning in fatigued metals Acta Meta. et Mat., vol. 43, issue 7, pp. 2849-2857, July 1995.

[11] Ercolessi F. A molecular dynamics primer[J]. Spring college in computational physics, ICTP, Trieste, June, 1997. (Book style)

[12] Y. Mishin, Interatomic Potentials for Metals, Handbook of Materials Modeling, Berlin, Springer., Chapter 2.2, pp. 459-478, 2005. (Book style)
[13] G.P. Purja Puna and Y. Mishin Development of an interatomic potential for the Ni-Al system, Phil. Mag., vol. 89, issue 34-36, pp. 3245-3267, 2009.

[14] M.H. Musazadeh, K. Dehghani Molecular dynamic simulation of crack propagation in nanocrystalline Ni containing different shapes and types of second phases, Computational Materials Science, vol. 50, issue 11, pp. 3075-3079, Oct.Nov. 2011.

[15] Y. Mishin, D. Farkas, M.J. Mehl, D.A. Papaconstantopoulos, Interatomic potentials for monoatomic metals from experimental data and ab initio calculations. Phys Rev B. vol. 59, issue 5, pp. 3393-3407, Feb. 1999.

[16] X.Y. Liu, F. Ercolessi, J. Adams Aluminium interatomic potential from density functional theory calculations with improved stacking fault energy, Model. Simul. Mater. Sci. Eng. vol. 12, pp. 665-670, 2004.

[17] H.W. Sheng, M.J. Kramer, A. Cadien, T. Fujita, M.W Chen, Highly optimized embedded-atom-method potentials for fourteen fcc metals, Phys. Rev. B, vol. 83, issue 13, pp. 134118, Apr. 2011.

[18] Paul White, Molecular dynamic modelling of fatigue crack growth in aluminium using LEFM boundary conditions, International Journal of Fatigue, vol. 44, pp. 141-150, Nov. 2012.

[19] Po-Hsien Sung, Tei-Chen Chen Studies of crack growth and propagation of single-crystal nickel by molecular dynamics, Computational Materials Science, vol. 102, pp. 151-158, May, 2015.

[20] Kai Zhou, Bin Liu, Yijun Yao, and Kun Zhong Effects of grain size and shape on mechanical properties of nanocrystalline copper investigated by molecular dynamics, Materials Science and Engineering: A, vol. 615, pp. 92-97, 6 Oct., 2014.

[21] M. Wen, X.J. Xu, Y. Omura, S. Fukuyama, K. Yokogawa Modeling of hydrogen embrittlement in single crystal $\mathrm{Ni}$, Computational Materials Science, vol. 30, issue 3-4, pp. 202-211, Aug., 2004.

[22] S. Boucetta, T. Chihi, B. Ghebouli, M. Fatmi, Firstprinciples study of the elastic and mechanical properties of $\mathrm{Ni}_{3} \mathrm{Al}$ under high pressure, Materials Science-Poland, vol. 28, No. 1, pp. 347-355, 2010.

[23] G. Brunetti, et. al. Determination of $\gamma-\gamma^{\prime}$ lattice misfit in a single-crystal nickel-based superalloy using convergent beam electron diffraction aided by finite element calculations, Micron, vol. 43, issue 2-3, pp. 396-406, Feb., 2012.

[24] A.S. Argon, PRECIPITATION STRENGTHENING, In Strengthening Mechanisms in Crystal Plasticity, by A.S. Argon, Oxford: Oxford University Press, 2007. Oxford Scholarship Online, 2007.

[25] Alexander Stukowski, Structure identification methods for atomistic simulations of crystalline materials, Modelling Simul. Mater. Sci. Eng., vol. 20, issue 4, pp. 45021, 2012.

[26] Helio Tsuzuki, Paulo S. Branicio, Jose P. Rino, Structural characterization of deformed crystals by analysis of common atomic neighborhood, Computer Physics Communications, vol. 177, issue 6, pp. 518-523, 15 Sep., 2007.

[27] Andre Pineau, Stephen D. Antolovich, High temperature fatigue of nickel-base superalloys A review with special emphasis on deformation modes and oxidation, Engineering Failure Analysis, vol. 16, pp. 2668-2697, 2009.

[28] Y. Mishin,Atomistic modeling of the $\gamma$ and $\gamma^{\prime}$-phases of the NiAl system, Acta Mater. vol. 52, issue 6, pp. 1451-1467, 2004.

[29] Y.S. Touloukian, R.K. Kirby, R.E. Taylor and P.D. Desai, Thermophysical Properties of Matter - the TPRC Data Series; Chapter 13 Thermal Expansion: Metallic Elements and Alloys, vol. 12, Plenum, New York, 1975. (Book style)

[30] Franz Aurenhammer, Voronoi Diagrams - A Survey of a Fundamental Geometric Data Structure, ACM Computing Surveys, vol. 23(3), pp. 345-405, 1991. 
[31] Mikael Segersäll, Daniel Leidermark, Johan J. Moverare, Influence of crystal orientation on the thermomechanical fatigue behaviour in a single-crystal superalloy, Materials Science \& Engineering A, vol. 623, pp. 68-77, 19 Jan., 2015.

[32] Y. Chen, G.F. Zhang, T.D. Jin, S.M. Wu, B. Peng, Quantitative modelling of electricity consumption using computational intelligence aided design, J. Clean. Prod. vol. 69 pp. 143-152, 2014.

[33] P. Beardmore, R. G. Davies, T. L. Johnston, On the temperature dependence of the flow stress of nickel-base alloys, Transactions of the Metallurgical Society of AIME, vol. 245, pp. 15371545, 1969.

[34] Y. Chen, B. Peng, Multi-objective optimization on multilayer configuration of cathode electrode for polymer electrolyte fuel cells via computational-intelligence-aided design and engineering framework, Applied Soft Computing, In Press, Accepted Manuscript, Available online 27 Feb., 2016, DOI: $10.1016 /$ j.asoc.2016.02.045.

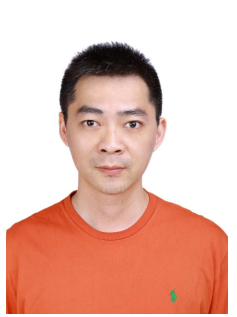

Jianfeng Huang who born in China, 1978 , now is a doctoral student of School of Engineering and Built Environment, Glasgow Caledonian University(GCU), Glasgow, Scotland, United Kingdom. His research subject is Computational Intelligence Based
Optimal Design of Superalloys. he acquired his Master degree of Material Processing and Engineering in 2004 from School of Materi-

al Engineering, Huazhong University of Science and Technology(HUST), Wuhan, China and the Bachelor degree of Mechanical Engineering in 2000 from Xiangtan University, Xiangtan, China. Before the study in Glasgow Caledonian University, he riched his working experience in CAD/CAE/CAM software development, especially in the pre-processing and postprocessing of dynamic simulation and FEM, in Siemens PLM Software from 2004 to 2014 as a senior software engineer.

During his study in HUST, he worked with staffs in State Key Laboratory of Material Processing and Die \& Mould Technology. He researched the key theory of the strip layout of progressive die and focused on the knowledge engineering application in the process of progressive die and proposed the hybrid knowledge representation method which integrates rule-based knowledge representation and case-based knowledge representation. He published a paper on the journal: Research on the Expression Methods of the Stamping Process Knowledge Based on KBE, Die \& Mould Industry, Vol 7, 2004.

Mr. Huang is a GCU scholarship recipient which covers his home tuitions for his $\mathrm{PhD}$ studies. And also he is a part of the Marie Skodowska-Curie Actions in Horizon 2020, Research and Innovation Staff Exchange (RISE) team.

E-mail: jianfeng.huang@gcu.ac.uk orcid.org: 0000-0001-98716303 